\title{
Estudios experimentales de la percepción desde la profesionalización de la Psicología en Argentina
}

\section{Estudos experimentais da percepção da profissionalização da Psicologia na Argentina}

\section{Experimental Studies on perception since Psychology's professionalization in Argentina}

\author{
María Andrea Piñeda* \\ Consejo Nacional de Investigaciones Científicas - CONICET / Universidad Nacional \\ de San Luis - UNSL, San Luis, Argentina
}

\author{
Patricia Scherman** \\ Universidad Nacional de Córdoba - UNC, Córdoba, Argentina
}

\begin{abstract}
RESUMO
O presente trabalho questiona o lugar que ocuparam os estudos experimentais sobre a percepção na Psicologia argentina, depois de sua profissionalização. Descreve-se três grupos que sistematicamente dedicaram-se ao tema entre a década de 1960 e a década de 1980: CIAL, LIS e UNCy/UNSL. São analisadas suas figuras principais e suas linhas de pesquisa. Indaga sobre o grau de coesão que alcançaram como comunidade científica nacional e, a partir de uma perspectiva policêntrica, descreve sua inserção internacional. Por fim, aborda aspectos institucionais e culturais próprios dos cursos de psicologia na Argentina que atuaram limitando o desenvolvimento dos estudos experimentais sobre a percepção.
\end{abstract}

Palavras-chave: psicologia experimental, percepção, laboratórios, universidade, Argentina.

\begin{abstract}
The aim of this paper is to analyze the place that experimental studies on perception had in Argentine Psychology after professionalization. Three research groups were identified to be devoted to perception studies during the period 1960's to 1980's: CIAL, LIS and UNCy/UNSL. Their leading figures and research streams are described. It is also inquired the cohesiveness these groups reached as a national scientific community. From a polycentric standpoint, their linkage to the international scientific community is also examined. Finally, some idiosyncratic institutional and cultural issues of Argentine Psychology Programs are discussed regarding the limitations of experimental studies on perception`s development.
\end{abstract}

Keywords: experimental psychology, perception, laboratories, universities, Argentine. 


\begin{abstract}
RESUMEN
Se indaga el lugar que ocuparon los estudios experimentales sobre la percepción en la psicología argentina tras la profesionalización de la psicología. Se describen los tres grupos que sistemáticamente se dedicaron a dicho tema desde la década de 1960 a la década de 1980: CIAL, LIS y UNCy/UNSL. Se examinan sus figuras principales y sus líneas de investigación. Se indaga el grado de cohesión que alcanzaron como comunidad científica nacional. Desde una perspectiva policéntrica, se describe su inserción internacional. Se discuten aspectos institucionales y culturales propios de las carreras de psicología argentinas que incidieron en la limitación del desarrollo de los estudios experimentales sobre la percepción.
\end{abstract}

Palabras clave: psicología experimental, percepción, laboratorios, universidad, Argentina.

\title{
1 Introducción
}

Los laboratorios psicológicos florecieron en Argentina desde la última década del siglo XIX, en el período inicial de la psicología, caracterizado por el interés en la psicología experimental, clínica y social (Klappenbach, 2006). Durante el segundo periodo, distinguido por el avance de la psicología filosófica (1916-1941), los laboratorios de psicología experimental no desaparecieron pero sus producciones quedaron asumidas en modelos totalistas. En el tercer período (19411962) en el que la psicotecnia y la orientación profesional tomaron un fuerte impulso, los aparatos de laboratorio más bien fueron desplazados por los tests mentales y la investigación cobró un carácter mayoritariamente aplicado. En el cuarto período (19621984), luego de creadas las carreras de psicología en las principales universidades del país, los debates se centraron en el rol profesional del psicólogo y la investigación psicológica fue desplazada de la escena académica (Klappenbach, 2006).

Así, las líneas de investigación experimental que se desarrollaban en Argentina a principios del siglo $X X$, formaban parte del proceso de inauguración de la nueva ciencia. La organización de los primeros laboratorios entre 1891 y 1905 por Víctor Mercante y Horacio Piñero respectivamente, sentaron las bases del estudio científico de las funciones mentales normales y desagregadas y de las aptitudes sensoriales y motoras, combinando procedimientos psicofísicos con aparatos de laboratorio y tests mentales. Estas tradiciones se continuaron en la Universidad Nacional de La Plata y la de Buenos Aires por algunas décadas (Falcone, 2012; Klappenbach, 2006; Papini, 1976; Talak, 2000, Vezzetti, 1988). También se ha destacado el trabajo realizado en la Facultad de Medicina de la Universidad Nacional de Córdoba por el fisiólogo italiano Virgilio Ducceschi (Ferrari, 2013). Ya en la segunda y tercera década, de gran originalidad han sido los trabajos de Christfried Jakob sobre 
psicofisiología cerebral (Triarhou y Del Cerro, 2006), Enrique Mouchet sobre lenguaje y pensamiento, percepción táctil en ciegos y emoción (Detrixhe \& Papini, 1978), Juan Cuatrecasas sobre psicofísica de los sentidos y percepción óptica (Carpintero, Ferrándiz \& Lafuente, 1997). Asimismo reconocemos a Alfredo Palacios, Carlos J esinghaus y Gregorio Fingermann en psicofisiología aplicada a la orientación profesional con los primeros estudios sobre la fatiga y accidentes de trabajo (Rossi, Ibarra \& Kirsch, 2010). No obstante, la psicología experimental argentina se vio significativamente limitada por ideales humanistas en el contexto de entreguerras mundiales y crisis políticas locales.

Al igual que otros países latinoamericanos (por ejemplo, Brasil), tras la Segunda Guerra Mundial, Argentina vivió un proceso de industrialización en el cual la psicotecnia cobró un importante impulso, fundamentalmente en el área de la educación y el trabajo (Piñeda \& Jacó-Vilela, 2014). Este proceso precipitó la profesionalización de la psicología a partir de 1955 con una impronta psicotécnica. Sin embargo, dicho modelo fue rápidamente desplazado por nuevas matrices inspiradas en intereses clínicos, en los que el existencialismo y luego el psicoanálisis prevalecieron como corrientes teóricas dominantes, incidiendo notablemente en la definición del rol del psicólogo en el que fue acentuándose un sesgo clínico (Klappenbach, 2006). En algunos casos, estos modelos se fueron integrando en una cultura humanista con un fuerte componente contestatario que rechazaba la psicología experimental por asociarla a intereses imperialistas norteamericanos. Estos factores se complementaron con el desfinanciamiento de la investigación científica en las universidades nacionales que desde 1966 se fue agravando tras cada golpe de Estado. Así, en un contexto general y progresivo de vaciamiento de la capacidad científica de las universidades, las carreras de psicología carecieron de financiamiento tecnológico para el montaje de laboratorios de psicología experimental. Los investigadores orientados a estas prácticas se fueron desplazando a institutos de Facultades de Medicina e Ingeniería donde encontraron esas estructuras ya consolidadas, o a instituciones extra-universitarias. Así, la psicología experimental fue perdiendo espacio en la formación de los psicólogos.

En contrapartida, otros factores promovieron el desarrollo de la investigación psicológica en una posición marginal a las carreras de psicología y a la profesión del psicólogo. En 1958 la creación del Consejo Nacional de Investigaciones Científicas y Técnicas (CONICET), organismo nacional de promoción científica, contribuyó decisivamente al desarrollo de la ciencia argentina y paulatinamente también hizo lugar al impulso de los estudios experimentales en psicología. Este organismo en sus orígenes estaba integrado por importantes especialistas de diferentes campos científicos, entre los 
que se destaca la figura del premio Nobel en Medicina, Dr. Bernardo Houssay. La promoción de la investigación científica incluyó el financiamiento de proyectos, del personal dedicado a la investigación -técnicos, becarios e investigadores-, de la creación de institutos de investigación y también el establecimiento de estándares científicos internacionales, cuya adopción promovió el establecimiento de una cultura científica de carácter internacionalista (Hurtado, 2010). La orientación del CONICET hacia el desarrollo de la ciencia básica, si bien significó inicialmente un contrapunto con las tendencias aplicadas desarrolladas por la psicología hasta el momento, contribuyó a sostener la investigación experimental. No obstante, la inclusión de las humanidades y de la psicología en el presupuesto del CONICET no se produjo desde el inicio. En efecto, las primeras becas de investigación fueron destinadas a las ciencias exactas y, luego de fuertes discusiones al interior del grupo fundador, se lograron establecer becas para sociología y psicología (Hurtado, 2010).

Tras este complejo proceso, las promisorias líneas de investigación experimental de la segunda y tercera década del siglo XX, entre las que se destacaron las de psicofísiología de la percepción que hemos mencionado, escasamente se incluían en el mapa de la investigación psicológica argentina de las décadas de 1960 a 1980 (Piñeda \& J acóVilela, 2014). En efecto, en este período en el que se modeló la profesionalización de la psicología en el país, sólo se han individualizado tres instituciones que sistemáticamente produjeron investigación experimental en psicología de la percepción: El Centro de Investigaciones Acústicas y Luminotécnicas ( $\mathrm{Cl} A \mathrm{~L}$ ) de la Facultad de Arquitectura y Urbanismo de la Universidad Nacional de Córdoba (UNC), el Laboratorio de Investigaciones Sensoriales (LIS) de la Facultad de Medicina de la Universidad de Buenos Aires (UBA) y, en San Luis, el Instituto de Investigaciones Psicopedagógicas de la Universidad Nacional de Cuyo (UNCy) que desde 1973 se transformó en la Universidad Nacional de San Luis (UNSL), en la que entre 1980 y 1983 funcionó un Laboratorio de Psicología Experimental.

Desde este punto de vista, los tres institutos cobran relevancia como casos testigo para el análisis de los factores que posibilitaron o limitaron el desarrollo de los estudios experimentales sobre la percepción en Argentina. Los tres centros han estado organizados en universidades nacionales, pero el tipo de vinculación de cada uno con las carreras de psicología ha sido diferente. En San Luis, el Instituto dependía de la carrera de psicología, en Córdoba el CIAL se organizó en la Facultad de Arquitectura pero con convenio con dicha carrera, y en Buenos Aires, el LIS ha sido completamente ajeno a la misma e incorporado al CONICET. Esta disparidad nos brinda una oportunidad para esbozar el papel que han jugado las variables académicoinstitucionales y las políticas científicas nacionales de financiamiento 
de esta línea de investigación en universidades nacionales e institutos de CONICET.

De modo que, en el presente trabajo nos proponemos la caracterización y comparación del desarrollo que las investigaciones en psicología de la percepción en el LIS, CIAL y UNCy/UNSL alcanzaron durante las décadas de 1960 y 1980, período en que se enfatizaron los debates por la profesión y el rol del psicólogo.

Para tales objetivos, nos basamos en el rastreo, análisis e interpretación de fuentes documentales. En este sentido, debemos advertir que las fuentes utilizadas no están estandarizadas sino que son dispares como las instituciones que son objeto de estudio, por cuanto no ha sido posible acceder a idéntico tipo de fuentes en las mismas, ya sea por motivos de conservación o porque los documentos producidos en cada caso no han seguido un patrón estándar. De modo que procuramos subsanar dicha limitación con la cantidad y variedad de fuentes complementarias que sirven a los objetivos planteados. En primer lugar, privilegiamos los documentos institucionales de los tres centros que aportan evidencias sobre sus objetivos y estructura, líneas de investigación, formación de recursos humanos y financiamiento (por ejemplo, informes de investigación y memorias institucionales, órdenes de compra, inventarios de equipamiento). En segundo lugar, para ahondar en el perfil académico de las figuras clave de cada equipo, analizamos notas autobiográficas o legajos y currículum vitae de los investigadores, así como algunos testimonios orales cuando nos resultó posible. Respecto del $\mathrm{ClAL}$, se analizó el legajo en CONICET y un relato autobiográfico de su fundador, el Ingeniero Guillermo Fuchs, y los curriculum vitae y testimonios orales de Cristina Biassoni y Ana María Verzini, las dos psicólogas que se iniciaron y permanecieron como personal del $\mathrm{ClAL}$ durante todo el período indagado. También complementamos con sus tesis de licenciatura y publicaciones realizadas en el $\mathrm{CIAL}$ en ese tiempo. En cuanto al LIS, nos hemos centrado en la persona de Miguelina Guirao, su fundadora, líder y única psicóloga del equipo interdisciplinario en el período, accediendo a diversos relatos autobiográficos y minuciosos informes anuales de investigación, así como a sus publicaciones y algunos programas de cursos de psicología dictados. Sobre la UNCy/UNSL, indagamos particularmente la labor de Claribel Morales de Barbenza, iniciadora de la línea de trabajo sobre psicoacústica en dicha institución a través de su curriculum vitae, testimonio oral, informes de investigación, publicaciones, su tesis doctoral y tesinas dirigidas en la temática.

Por otra parte, nos interesa indagar el grado de cohesión que estos tres equipos de investigación guardaron entre sí como comunidad científica, y en qué medida reconocían alguna continuidad con las líneas de investigación en psicofisiología desarrolladas en Argentina en la segunda y tercera década del siglo XX. Las memorias de 
actividades institucionales en las que quedaron constancia de visitas, conferencias, proyectos de cooperación, etc. abrieron una vía para establecer la cohesión de los grupos, visión que complementamos con el análisis de las citas en sus publicaciones y tesis. A su vez, esto también resultó una vía para la indagación de las referencias a líneas de investigación más antiguas.

Finalmente, es necesario advertir que si bien la presente podría ser considerada una "historia local" de interés para el contexto argentino, nuestro análisis historiográfico de las instituciones en estudio se orientará a establecer algunas relaciones de las mismas con el contexto internacional. Inclinándonos por un enfoque policéntrico (Brock, 2014) que permite destacar tanto la influencia internacional mutua entre los centros en estudio como la existencia de diferencias locales, trataremos de caracterizar algunas relaciones de poder, prejuicios, resistencias y barreras culturales en estos procesos. En este sentido, cobra relevancia el análisis de datos como viajes de estudio, becas, compra de instrumentos, traducciones, conferencias, co-autorías, etc.

\section{La psicofísica argentina de mediados de siglo $X X$. Laboratorios, centros e institutos}

En el año 1956, en un marco general de renovación del sistema científico y de educación superior argentino (Buchbinder 2005), se creó el CIAL. Fue fundado por el Ingeniero Guillermo Fuchs, pionero de la ciencia acústica argentina. Inicialmente el ClAL se organizó en tres departamentos: Control de Ruidos, Acústica Arquitectónica y Electroacústica y Luminotecnia. En 1965 se incorporó el Departamento de Psicofísica, para el estudio de las reacciones subjetivas ante la estimulación física (Fuchs, 1969).

El equipo estuvo integrado por psicólogos, ingenieros y arquitectos porque se concebía la acústica desde una perspectiva integral, plural e interdisciplinaria en la que se resaltaba la importancia del estudio del ser humano como receptor y efector del estímulo físico, "como integrador de las sensaciones generadas por los estímulos exteriores" (Fuchs, 1969, p. 13), donde tanto el aspecto físico como psicológico completaban la comprensión del fenómeno. La carrera de psicología se había iniciado en 1958 en la UNC. Así, explorando nuevas posibilidades, el CIAL convocó a alumnos de grado de la carrera de psicología a realizar su trabajo final de tesis en psicoacústica. Esta resultó una plaza atractiva para estudiantes que pretendían explorar nuevos campos que en su plan de formación de grado no estaba contemplado. Dos de las estudiantes que completaron de este modo su formación de grado hacia fines de 1960, Cristina Biassoni y Ana María Verzini, permanecieron en el CIAL desarrollando esta línea de 
investigación. En la década de 1970 el ClAL fue desarrollando diferentes proyectos de investigación en psicología ambiental, vinculados a la exposición a ruidos industriales y urbanos, y también su influencia en el aprendizaje escolar (Murat, Verzini de Romera, Biassoni de Serra \& Fuchs, 1979). A partir de la década de 1980 se orientaron a la construcción de sus propios instrumentos de medición, tales como escalas de diferencial semántico, de actitudes hacia el ruido, y cuestionarios de contaminación sonora ambiental, a la vez que se inician los estudios sobre ecolocación (Verzini de Romera, Suárez de Bonet \& Biassoni de Serra, 1983; Verzini de Romera \& Suárez de Bonet, 1985).

Por su parte, el LIS fue organizado en 1968 por la doctora Miguelina Guirao. Graduada en Filosofía en la UBA en 1952, se doctoró en Psicología Experimental en Milán bajo la dirección de Agostino Gemelli antes de 1959 (Guirao, 2008). Guirao tuvo un breve pasaje por el ámbito de la psicología académica antes de la organización del LIS. Primero, fue miembro del Departamento de Orientación Vocacional dirigido por Nuria Cortada en la Universidad de Buenos Aires (Cortada, 2008) y luego, en 1960, dictó los cursos de Psicología Aplicada y de Orientación y Selección Profesional en la recientemente creada carrera de psicología de Rosario, como consta en los programas encontrados de dichos cursos (Universidad Nacional del Litoral, 1960a; 1960b). Pero, varios hechos parecen haberla orientado hacia otros rumbos. Guirao ha relatado que, tras su doctorado en Milán se interesó por la obra de Stanley Smith Stevens (1906-1973) (Teghtsoonian, 2001), particularmente el famoso texto Handbook of Experimental Psychology (Guirao, 2008). A partir de ese interés se entrevistó con Bernardo Houssay, Presidente de CONICET, quien le recomendó completar sus estudios desde la perspectiva sensorial. Entre 1962 y 1966, con Beca del CONICET, estudió en el Laboratorio de Psicofísica de la Universidad de Harvard (USA), dirigido por Stevens (Guirao, 2006). En 1966 el CONICET la incorporó como Investigadora Superior. En 1968 organizó el LIS en la Facultad de Ingeniería de la UBA, integrándose también como Profesora Adjunta de Psicoacústica. EI LIS pasó a depender de la Facultad de Medicina en 1971 y desde ese momento fue oficialmente incorporado al CONICET.

En la primera década del LIS, Guirao logró constituir un talentoso equipo interdisciplinario: arquitectos, ingenieros, fonoaudiólogos y médicos, varios de ellos investigadores de carrera, además de 17 becarios y 10 técnicos de personal de apoyo del CONICET (Laboratorio de Investigaciones Sensoriales, 1978).

El Laboratorio de Psicología Experimental se organizó en la UNSL entre 1980 y 1983, bajo la dirección de la Doctora Claribel Morales de Barbenza. Su antecedente directo fue el Instituto de Investigaciones Psicopedagógicas de la UNCy (1967-1972), dirigido primero por el Dr. 
Plácido Horas y luego por la Dra. Eva Mikusinski. La Doctora Barbenza, graduada como Profesora de Psicología y Pedagogía en 1958 en la UNCy y Licenciada en Psicología en 1966 en la misma institución, fue miembro del equipo de Investigación del Instituto de Investigaciones Psicopedagógicas, docente e investigadora de la carrera de psicología desde su graduación hasta su jubilación y finalmente Profesora Emérita en 1997. Entre 1967 y 1968 realizó una estancia de estudios doctorales en Londres para especializarse en los enfoques experimentales para el estudio de la psicoacústica (Individual Loudness Functions), allí obtuvo su Master of Sciences. Al año siguiente, defendió en San Luis su tesis doctoral: Funciones Individuales de Sonía (Universidad Nacional de Cuyo, Resolución $\mathrm{N}^{\circ}$ 1072/1969). La tesis, asesorada en San Luis por Plácido Horas, en Londres fue dirigida por William Tempest y la colaboración de M. E. Bryan del Departamento de Ingeniería, Unidad de Audiología, de la Universidad de Salford. En este trabajo, Barbenza se ocupaba de estudiar la intensidad subjetiva del sonido, tema promisorio por sus aplicaciones prácticas.

Las líneas de trabajo específicas diferían en cada institución. Respecto del $\mathrm{CIAL}$, hemos podido reconstruir (Biassoni y Verzini, comunicación personal, 3 de julio de 2015) el listado de tesis de licenciatura en psicología allí realizadas (Biassoni, 1968; Blanco, 1968; Castro de Ortiz, s/f; Cornachione, 1965; Fernández, 1967; Foschi, 1968; Orrico de Stuart, 1966; Saal, 1968; Saforcada, 1963; Trotanesky, S., 1965; Verzini, 1968; Yuzuk, 1967). Algunas de estas tesis aún se conservan permitiendo su análisis de contenido y han brindado indicios de los temas abordados y enfoques metodológicos. En general, se realizaron estudios de percepción auditiva y visual desde enfoques psicofísicos, psicolingüísticos y piagetianos con diseño empírico, pruebas experimentales y control de variables en las distintas cámaras del $\mathrm{ClAL}$. Una de las investigaciones (Saal, 1968) se realizó en una escuela, ya que incluía niños y adolescentes en su muestra.

Las perspectivas teóricas iniciales eran variadas e involucraban diferentes aspectos de la percepción. Del estudio de las referencias bibliográficas de las tesinas, hemos clasificado tres grupos de citas. Primero, las referencias presentes en todos los trabajos, entre los que encontramos dos textos en español: Fenomenología de la percepción de Merleau Ponty (1945), edición del Fondo de Cultura Económica y el manual de Woodworth \& Schlosberg (1964), Psicología Experimental, publicado por Editorial Eudeba. Segundo, identificamos autores que se encuentran en más de una de las investigaciones analizadas, donde aparecen diversos textos de Fuchs sobre acústica (1958, 1966), también de Piaget (1960), Piaget \& Inhelder (1947), el texto de Siegel (1956) sobre estadística no paramétrica en ciencias del comportamiento y también la obra de Chauchard (1953) Mensajes de nuestros sentidos. Finalmente, las referencias que aparecen en 
alguno de los trabajos, como Mira y Lopez (1952), Cuatrecasas (1963), Delgado \& I bérico (1966), Houssay (1955), Piéron (1960) y Blalock (1966), entre otros.

Respecto de los dos primeros textos, Fenomenología de la percepción y Psicología Experimental, advertimos que fueron utilizados en su versión en español, reafirmando la importancia de las editoriales locales en la difusión de perspectivas teóricas (Klappenbach, 2001). Las referencias comunes a todas las tesinas constituyen una superficie básica de recepción teórica de los miembros de la comunidad. En el listado de autores citados descubrimos aquellos locales y contemporáneos a ese contexto (por ejemplo: Houssay, Fuchs), que sintetizaban diversas tradiciones científicas extranjeras que circularon en Argentina tras la primera psicofísica. También registramos autores extranjeros que se recepcionaron en el período de auge de la psicotecnia y la psicología filosófica. Entre los más característicos, registramos las tradiciones francoparlantes en su versión fenomenológica (Merleau Ponty) y experimental (Pieron, Chauchard, Piaget), ambas muy difundidas en las carreras de psicología, y las últimas cumpliendo un rol decisivo en la implantación de la psicotecnia y en la cultura científica de los inicios de las carreras de psicología argentina. También encontramos la obra del español Mira y López (Rossi, Falcone \& Ibarra, 2014). Estas perspectivas europeas se citaban a la par de otras de procedencia norteamericana, como S. S. Stevens. De modo que, las tesis de grado analizadas son una fotografía de la interfase entre el humanismo y la psicotecnia de las décadas de 1940 y 1950 aún vigentes en los inicios de las carreras de psicología y por lo tanto comunes en la formación de los psicólogos de la época, y los nuevos enfoques experimentales recepcionados en el $\mathrm{ClAL}$. Pero no registramos referencias a las líneas de investigación psicofisiológicas de las décadas anteriores. En las publicaciones de Biassoni y Verzini ya graduadas, se observa preponderancia de referencias a autores norteamericanos de enfoques experimentales, particularmente a S. S. Stevens.

El equipo del LIS se ha ocupado de los procesos sensoriales como fundamento de la comunicación humana en sus aspectos neurofisiológicos, el código neural y los correlatos psicofísicos. Del análisis de sus memorias institucionales podemos descubrir sus líneas de investigación, así como se menciona el centenar de artículos que Guirao publicó en revistas científicas internacionales especializadas en los distintos sentidos, psicofísica, fisiología, psicolingüística, cibernética, incluyendo artículos en la Revista Science, que lograron gran impacto. Curiosamente, pocos de ellos aparecieron en revistas de psicología, como los tempranamente publicados con su mentor S. S. Stevens en la Journal of Experimental Psychology, o los de Perception and Psychophysics (Stevens \& Guirao, 1963; Stevens, Guirao \& Slawson, 1965; Stevens \& Guirao, 1967; Guirao \& 
Valciukas, 1975). Asimismo, en la obra de Guirao se destaca su libro: Los Sentidos, Bases de la Percepción, originalmente editado en Madrid por Alhambra en 1980, traducido a varios idiomas, y utilizado como texto en diversas cátedras de Europa y Latinoamérica. Es notable cómo, ya consolidado su camino como investigadora después de tres décadas, a la par de las citas a los mayores referentes de la moderna psicofísica, Guirao (1980) mantenía las referencias a su maestro Gemelli, con quien dio sus primeros pasos en la psicología experimental.

En suma, en el LIS el enfoque ha sido interdisciplinario incluyendo modelos biológicos y matemáticos, técnicas electrofisiológicas y técnicas psicofísicas, y los trabajos han utilizado metodologías eminentemente experimentales en tres laboratorios: 1) Fonética y Audio, 2) Óptica, 3) Bioquímica. En el primero, el estudio se centraba en el análisis del espectro acústico de la audición y de los sonidos del habla mediante instrumental electrónico, así como análisis del espectro característico del habla española; áreas acústicas de las vocales españolas y comparaciones con inglés y japonés. Respecto del estudio de la visión, los temas indagados incluían: capacidad de resolución del ojo humano, aspectos de la conducta regulados por factores visuales y variables que intervienen en dichos procesos. En cuanto al laboratorio de química, encontramos estudios neurofisiológicos del sistema olfatorio, análisis de las cualidades sensoriales de los alimentos con técnicas psicofísicas, capacidad de identificación de olores en personas normales y con trastornos en los sentidos químicos (Laboratorio de Investigaciones Sensoriales, 1978). Por tanto, si bien Guirao se formó en la matriz humanista de la UBA de mediados de siglo $X X$, en su primera formación de posgrado se observó un viraje hacia enfoques experimentales europeos aún compatibles con dicha matriz, que siguieron vertebrando su concepción integral del hombre y del campo disciplinar tras su segunda formación de posgrado orientada hacia modelos norteamericanos.

Mientras tanto, en San Luis Barbenza continuó trabajando sobre intensidad subjetiva del sonido. Al respecto, el término inglés loudness, utilizado en su trabajo original, en español admite diversas traducciones. Así por ejemplo Barbenza recogía en su tesis el término "sonoridad" dado por el Diccionario de Psicología de Warren, pero prefería el de "sonía" según la traducción castellana del término registrado en Vocabulaire de la psychologie de Piéron. Este autor había tenido gran incidencia en la cultura psicológica científica de mediados del siglo XX en Argentina, y en particular en la formación de los primeros psicólogos en San Luis, de acuerdo a la impronta marcada por el organizador de la carrera, Plácido Horas. En su tesis Barbenza proponía el estudio de la percepción en el contexto de la personalidad y las diferencias individuales. Desde una concepción 
totalista de la personalidad, se oponía a toda visión atomista y meramente empirista de la sensación. Valoraba la unidad, totalidad y significación de la experiencia perceptual en el procesamiento de estímulos extero y propioceptivos como resultado de una integración de imágenes mnémicas, afectos y motivaciones del sujeto percipiente. En este sentido, integraba diversos enfoques entre los que destacaba la Gestalt. En el estudio de las diferencias individuales, incorporaba la tradición británica desde Galton hasta Eysenck. Este último autor había sido tempranamente bien conocido en San Luis gracias a la introducción que la colega de Barbenza, Eva Mikusinski, hizo de Eysenck en San Luis y en el país.

Barbenza señalaba críticamente que, en general, los estudios experimentales sobre la percepción han tendido a ignorar las diferencias individuales por la dificultad metodológica que entrañan dichas variables, y que más bien los estudios se han centrado en la obtención de valores paramétricos en la búsqueda de conquistas técnicas (por ejemplo, escalas de sonía para las telecomunicaciones). En dicho contexto, Barbenza introducía el aporte de los ingleses Mc Robert, Bryan y Tempest (1965), en cuyo equipo de trabajo ella había desarrollado sus investigaciones. Estos abrían cierta controversia en relación a S.S. Stevens, también ampliamente citado en su tesis, pues señalaban que la sonía sometida al juicio por parte de observadores no era absoluta, sino relativa a variables de personalidad. En esa dirección, Barbenza notaba las contribuciones de diversos autores, entre las que destacamos las de J.C. Stevens \& M. Guirao (1964) que sugerían la incidencia de variables de personalidad al registrar la respuesta estable de los sujetos en diversas sesiones.

Más allá del contrapunto establecido entre las líneas de investigación de Harvard y Salford, queda claro que la elección del enfoque inglés de parte de Barbenza resultaba más acorde a la cultura científica argentina, por dar lugar a la consideración de la personalidad total como eje del estudio. En efecto, en los grupos académicos dominantes de la psicología argentina, la psicología experimental era resistida por atribuírsele connotaciones ideológicas adversas. Por otro lado, en San Luis, donde peculiarmente se había ido construyendo una psicología entendida como ciencia del comportamiento el estudio de la personalidad total era el campo principal de integración de pluralidad de enfoques teórico-metodológicos y el tronco común de desarrollo de las diversas áreas disciplinares. En San Luis, durante el pasado inmediato a los estudios de Barbenza, las preferencias hacia las tradiciones europeas se habían evidenciado mayormente hacia la psicología experimental francesa, que parecían ser garantía del estudio global y al mismo tiempo riguroso del comportamiento. Signos de esa cultura científica emergían en las citaciones de Barbenza a Piéron y a Fraisse, donde la integración con la tradición de la década de 1950 fue significativa, en tanto que el viraje hacia 
enfoques norteamericanos fue menor que el registrado en el $\mathrm{ClAL}$ y el LIS. En definitiva, las líneas de trabajo que Barbenza había abierto con sus estudios en Inglaterra se integraban a la cultura académica de la carrera de psicología de San Luis sin mayores resistencias. Esto no hubiera sido posible en las carreras de psicología de la UBA o de la UNC donde el sesgo clínico y psicoanalítico era proporcional a las altas resistencias a los enfoques experimentales, razón por la cuál sólo pudieron desarrollarse en organismos ajenos a las mismas como el LIS o el CIAL.

\section{Formación de recursos y vinculaciones internacionales}

El diseño curricular que formaba psicólogos en la UNC, al igual que en el resto del país, no ponía especial énfasis en la psicología experimental, sino que predominaba un marcado sesgo clínico. Sin embargo, es preciso señalar que, desde sus inicios, los directores de la carrera de psicología realizaron diversos intentos de fortalecer el perfil científico de la misma. Por un lado, como ya indicamos, las tesinas de grado concebidas como trabajos de investigación empíricos constituyeron una pieza clave en la formación de recursos humanos especializados en la UNC ya que actuaron como un contrapeso frente a la formación clínica y psicoanalítica predominante, permitiendo otro tipo de elección profesional. Por otra parte, docentes de estadística, metodología y psicometría, tales como Rimoldi, Fogliatto y Murat, mantuvieron activos vínculos con el $\mathrm{ClAL}$, asesorando investigaciones, dictando cursos, asesorando tesinas e incluso participando de algunas investigaciones, como en el caso de Murat (Murat, Verzini de Romera, Biassoni de Serra \& Fuchs, 1979). En relación a la psicoacústica, estudiantes y graduados debieron capacitarse para la utilización del instrumental necesario para llevar adelante los estudios sobre el aspecto físico del sonido (Verzini y Biassoni, comunicación personal, 6 de noviembre de 2013). Ese fue el caso de Biassoni y Verzini, las psicólogas incorporadas a la investigación en el $\mathrm{ClAL}$, que ya graduadas y alejadas de la carrera de psicología, siguieron formándose en el $\mathrm{ClAL}$ con referentes internacionales con los que realizaron numerosos intercambios. Por ejemplo, se tomaron cursos con J. C. Stevens de la Universidad de Yale, USA y Guirao en el LIS, en 1969; con John Walker en 1972 y Alan Martin en 1973, del Institute of Sound and Vibration Research (Southampton University, Inglaterra); Douglas Muster (Universidad de Huston, Texas, USA) también en 1973. En el año 1965 el ClAL fue sede de las 1 ㅇ Jornadas Latinoamericanas de Acústica, donde se promovió la creación del Grupo de Acústicos Latinoamericanos (GALA) del que Fuchs fue su primer Presidente (Serra, 2000). La 
Jornada tuvo su segunda edición en Córdoba en 1972, siendo ya el $\mathrm{CIAL}$ en una de las principales referencias latinoamericanas.

Entre 1965 y 1981, el ClAL contó con la visita de grandes figuras internacionales como la del Premio Nobel en Medicina Von Békesy, y a Eberhard Zwicker, y también otras personalidades de Estados Unidos, España e Inglaterra ya mencionadas.

El Seminario Latinoamericano de Acústica organizado por el $\mathrm{ClAL}$, de marzo a abril de 1982, bajo el patrocinio de la Organización de los Estados Americanos (OEA), nos permite advertir el progreso de sus investigaciones y la intensidad con que se impulsaban los contactos de cooperación internacional especialmente con centros de Alemania, Estados Unidos, Japón y China.

También el LIS contó con la visita de numerosos y destacados investigadores de diversas latitudes, en el marco de un estable sistema de intercambio científico para dictado de cursos y asesoramiento de investigaciones. En 1968 también recibió a Georg von Békesy a quien Guirao había conocido en Harvard. Von Békesy fue invitado a dictar un curso de posgrado sobre Biofísica de los Sentidos, del que también participaron miembros del ClAL (Biassoni, 2013). Además, él recibió el Doctorado Honoris Causa en la UBA y en la UNC, verificándose así relaciones entre Guirao y el ClAL. Este vínculo interinstitucional, lejos de ser ocasional, se sostuvo en diversos eventos documentados, como por ejemplo la conferencia "Cibernética y Sociedad: Homenaje a Norbert Wiener, organizada conjuntamente con la Secretaría de Extensión Universitaria de la UNC (Laboratorio Investigaciones Sensoriales, 1978). Otro de los investigadores de primera línea frecuentemente invitados al LIS fue J. C. Stevens (USA), co-autor de Guirao que en ese período los visitó tres veces.

Al mismo tiempo, Guirao y su equipo dictaron cursos en el exterior. Entre ellos: Universidad de Harvard y Columbia (USA); City University of London (Inglaterra), Universidades de Tokio, Kioto, Osaka y Saporo (Japón), diversos centros de investigación de China, Universidad de Campinas (Brasil). En la psicología latinoamericana existía una comunidad académica dedicada a la percepción que la reconocía como un referente en la materia. Así por ejemplo, José Aparecido Da Silva la convocó junto a miembros de su equipo y a investigadores de Brasil, Estados Unidos, Italia, Japón para publicar un número especial de la Revista Latinoamericana en Psicología dirigida por Rubén Ardila- dedicado a la psicofísica y psicología de la percepción (Guirao, 1987).

Por su parte, Barbenza mantuvo los vínculos con su equipo de trabajo en Inglaterra durante la década del setenta así como su co-autoría con ellos en revistas internacionales interdisciplinarias (Bryan, Tempest \& Barbenza, 1969; Barbenza, Bryan \& Tempest, 1970; Barbenza, Bryan, Tempest \& McRobert, 1970) y también publicó sus 
trabajos en medios latinoamericanos y españoles (Barbenza, 1974; Barbenza, 1976; Barbenza \& Uhrlandt, 1983). En San Luis se llevaron a cabo dos proyectos específicos en psicoacústica entre 1979 y 1984, que fueron financiados por la Secretaría de Ciencia y Técnica de la UNSL: Efectos del ruido sobre el organismo humano y la conducta (1979-1982) y Sensibilidad al ruido y parámetros de personalidad (1983-1984) (Universidad Nacional de San Luis, 1982). Si bien, después de ese período Barbenza continuó trabajando sobre procesos psíquicos básicos (Barbenza, 1992), se orientó cada vez más a temas de evaluación de la personalidad mediante el MMPI y otros tests mentales, algo perfectamente acorde con la cultura académica y el presupuesto de las carreras de psicología argentinas.

Pero estos vínculos internacionales en el campo de la psicoacústica no mantuvieron su incidencia en la formación de psicólogos en San Luis. La línea de investigación iniciada por Barbenza no alcanzó a consolidarse en el tiempo. Después del Golpe de Estado de 1976 y aún tras la recuperación democrática de 1983 no se logró sostener la política de formación de recursos humanos en el exterior y la contratación de docentes extranjeros impulsada por Plácido Horas en las décadas de 1960 y principios de 1970 que le había permitido a Barbenza formarse en Inglaterra. Por tanto, no pudo consolidarse un equipo de investigadores especializados en este campo. En efecto, en esta temática registramos solo cuatro tesis de grado entre 1976 y 1977 bajo la dirección de Barbenza. Los investigadores que al graduarse continuaron trabajando en su equipo, se orientaron al estudio experimental del comportamiento en un sentido amplio, y solo una de ellas publicó algunos artículos sobre psicoacústica en coautoría con Barbenza hasta mediados de la década de 1980 (Barbenza \& Uhrlandt, 1983).

Como hemos señalado, Barbenza conocía los trabajos de Guirao previos a su establecimiento en Argentina en el LIS y los citó en su tesis de Doctorado. Por otra parte, hemos comprobado que después de dicha formación Barbenza también mantuvo vínculos con el $\mathrm{ClAL}$ para asesoramiento y evaluación de proyectos (Biassoni y Verzini, Comunicación personal 6 de noviembre de 2013). Por su parte, ya explicamos cómo el ClAL también estaba activamente vinculado al LIS y el trabajo de Guirao, y referenciaban a S. S. Stevens. Con lo cual, queda claro que había cierta cohesión entre los tres centros estudiados, y todos ellos se orientaban hacia el epicentro de la psicofísica de los sentidos y la percepción de la época.

\section{El fomento a la investigación: el papel del Conicet y otras instituciones financiadoras}


En el $\mathrm{ClAL}$ el equipamiento necesario para el desarrollo de investigaciones se financiaba con fondos del CONICET, del Gobierno Británico y de Laboratorios Nacionales de Investigación y Servicios (LANAIS) (Biassoni y Verzini, comunicación personal, 6 de noviembre de 2013). Si bien el CIAL no pertenecía al CONICET, su director era Investigador de dicho organismo, llegando a la categoría de Investigador Superior, la máxima del sistema científico argentino (Consejo Nacional de Investigaciones Científicas y Técnicas, s/f). También los investigadores eran financiados por CONICET, como fue el caso de Biassoni y Verzini. Se advierte aquí el papel del CONICET en la sustentabilidad de las investigaciones y la promoción de la temática.

Logrando el LIS incorporarse al CONICET, fue este organismo el que establemente financió los recursos humanos y materiales (Laboratorio Investigaciones Sensoriales, 1978). Además de moderno equipamiento, el LIS contaba con una nutrida biblioteca, hemeroteca y videoteca especializada en ingeniería, biología, ciencias sociales y del comportamiento, en épocas en que obtener bibliografía internacional y suscripciones periódicas en Argentina no era sencillo.

En San Luis, la formación de recursos humanos para la investigación, y la investigación misma, durante la década de 1960 estuvo financiada por la Secretaría de Ciencia y Técnica de dicha universidad y en alguna medida por subsidios de la OEA y algunas Becas de CONICET, pero en escasas situaciones, se obtuvo un financiamiento más o menos sostenido, como fue el caso de Eva Mikusinski. En San Luis no llegó a constituirse un Instituto de CONICET que asegurara el financiamiento sostenido y la estabilidad institucional necesaria para el desarrollo de la psicología experimental y la psicoacústica que la carrera de psicología no podía ofrecer. De hecho, el Laboratorio de Psicología Experimental creado por Barbenza en 1980 (Universidad Nacional de San Luis, Ord. 05/1980), tuvo corta vida. Recién estuvieron dadas las condiciones para revitalizar este proyecto en 1990 cuando Barbenza organizó el Laboratorio de Investigaciones en Ciencias del Comportamiento. No obstante, esta fragilidad institucional a inicios de 1980, la comunidad académica parecía haber logrado la suficiente cohesión como para institucionalizar un modelo de psicología alternativo al más difundido en las carreras de psicología argentinas. Tras el proceso de recuperación democrática y de normalización de las universidades, San Luis fue sede en 1987 de la creación de la Asociación Argentina de Ciencias del Comportamiento (AACC) y Claribel Barbenza fue su primera Presidente. Esta Asociación, hoy representante de Argentina ante la IUPsys, tuvo el mérito de reunir psicólogos dedicados a las ciencias del comportamiento que no tuvieron cabida en las carreras de psicología, así como investigadores de otros campos afines de diversas instituciones. 


\section{Discusión}

Procuramos analizar el lugar que ocuparon los estudios experimentales sobre la percepción en la psicología argentina tras la profesionalización de la psicología describiendo los tres grupos que sistemáticamente se dedicaron a ello. En sus líneas de trabajo no parecía haber vestigio de referencias a la psicofísica de las primeras décadas del siglo XX. En cambio, registramos enfoques característicos de la psicología argentina de las décadas de 1940 y 1950 (por ejemplo, antropologías filosóficas orientadas hacia la fenomenología; referencias a la psicotecnia y a la psicología experimental francoparlante) que se expandieron en una cultura académica humanista que incidió en las matrices de organización de las carreras de psicología. Desde aquí, se fueron aceptando nuevas tradiciones anglosajonas, siendo más notoria en el LIS y en el CIAL la elección del modelo de S. S. Stevens, de Harvard, que en UNCy/UNSL donde la balanza se inclinó por el modelo de Tempest, de Salford, que parecía responder mejor a las expectativas de una cultura académica consolidada en la matriz comportamental de la personalidad total.

Establecimos relaciones de cohesión entre los tres grupos de investigación que pudieron comprobarse por diversas vías. Más allá de las referencias a la antropología filosófica y a la psicotecnia argentina de las décadas de 1940 y 1950 que los tres grupos poseían como plataforma cultural, todos citaban a S. S. Stevens como referente ineludible de los estudios experimentales de la percepción. De la misma manera, su discípula Guirao se había convertido en un referente nacional. Barbenza la citaba en su tesis doctoral; Biassoni y Verzini tomaron cursos de posgrado con Guirao; el LIS y el CIAL mantuvieron relaciones de colaboración para la formación de recursos humanos de posgrado y la organización de reuniones científicas (por ejemplo, el otorgamiento del Doctorado Honoris Causa de Von Béckesy en la UNC), y Barbenza asesoraba investigaciones en el $\mathrm{ClAL}$.

Al mismo tiempo, se pudo determinar que los tres grupos han tenido importante inserción en la comunidad internacional especialista en percepción. Sin dudas, las relaciones internacionales del $\mathrm{ClAL}$ han sido muy prominentes, alcanzando liderazgo en Latinoamérica mediante GALA. Las del LIS han sido descollantes, proyectándose a Estados Unidos, Europa y Asia, logrando invertir en alguna medida las tradicionales relaciones de recepción en el desarrollo disciplinar, pasando a ser productor activo en el conocimiento de primera línea. Por su parte, la inserción de la UNCy/UNSL en la comunidad internacional ha sido más limitada en el alcance y en el tiempo, por 
no lograr el apoyo político-económico que le diera suficiente fuerza institucionalizante.

Sugerimos que las diferencias de desarrollo entre las tres instituciones estudiadas en primer lugar guardaron relación con las políticas de promoción de ciencia y tecnología que pudo gestionar cada una. En segundo lugar, esto estuvo íntimamente ligado al financiamiento que el Estado Nacional les otorgó en función de sus políticas globales de desarrollo de ciencia y tecnología, con débil apoyo en las universidades y de mayor contundencia en la estructura del CONICET. En efecto, la promoción de la investigación científica experimental fue escasa en el contexto de las carreras de psicología. Por otra parte, debido a la cultura institucional humanista, sesgada hacia la clínica psicoanalítica que predominaba en estas, se hizo necesario establecer lazos con otras unidades académicas para promover la investigación experimental. Esto se logró por varias vías, a veces complementarias. El convenio con otras Facultades de la misma universidad con mayor financiamiento para el desarrollo de ciencia básica y aplicada (como el ClAL), o el total desplazamiento a otras Facultades e institutos de CONICET sin relación con las carreras de psicología (como el LIS). La formación en el exterior del país (los casos de Guirao y Barbenza), o el establecimiento de vínculos de cooperación internacional para la formación de recursos humanos y el asesoramiento de proyectos (como el LIS y el CIAL) para garantizar la permanencia de estas líneas de desarrollo.

En un país donde la investigación científica no cuenta con tradición de financiamiento privado, las investigaciones que lograron sostenerse en el tiempo y alcanzaron mayor relieve internacional se financiaron por el CONICET. El apoyo más sostenido de este organismo se logró en el marco de Facultades ajenas a las carreras de psicología, principalmente en aquellas vinculadas al campo de la Medicina y la Ingeniería, campos que dominaron el CONICET desde su creación. Dicha forma de institucionalización de la investigación experimental acaso haya tenido el costo de que Guirao perdiera su identificación como psicóloga, y Biassoni y Verzini se alejaran de la formación de psicólogos. Esta disociación entre investigación experimental y carreras de psicología siguió reforzando la reproducción de la matriz humanista y psicoanalítica, que en primer lugar había obligado a la psicología experimental de la percepción a buscar mejores posibilidades de desarrollo en otros organismos. La disociación de la formación de los psicólogos del perfil de investigador experimental, si bien no discontinuó estas prácticas, seguramente contribuyó a perder este campo de las competencias del psicólogo argentino.

Finalmente, resulta llamativo que las figuras que hemos analizado en los tres centros han sido mujeres abriéndose camino en contextos institucionales gobernados por hombres. Psicólogas, cuya profesión 
en Argentina ha tenido un predominio femenino, buscaron su lugar en campos culturalmente ganados por ingenieros y médicos.

\section{Referencias}

Barbenza, C. (1974). Efectos de drogas excitantes y depresoras sobre las funciones de intensidad subjetiva del sonido. Revista Latinoamericana de Psicología, 6(3), 49-58.

Barbenza, C. (1976). Funciones de sonoridad, personalidad y drogas. Revista Latinoamericana de Psicología, 8(1), 283-293.

Barbenza, C. (1992). Curriculum Vitae. Legajo de Claribel Barbenza en la Universidad Nacional de San Luis. Mimeo.

Barbenza, C., Bryan, M. E. \& Tempest, W. (1970). Individual Loudness Functions. Journal of Sound and Vibration, 11(4), 339-410.

Barbenza, C., Bryan, M. E., Tempest, W. \& McRobert, H. (1970). Individual loudness susceptibility. Sound, 4, 75-79.

Barbenza, C. \& Uhrlandt, M. (1983). Algunos efectos del ruido sobre el organismo humano y la conducta. Revista de Psicología General y Aplicada, 36(5), 867-880.

Biassoni, C. (1968). Juicios subjetivos sobre fuerza sonora y molestia de ruidos domésticos y su relación con medidas físicas de aislación. Tesis de licenciatura no publicada. Córdoba: Universidad Nacional de Córdoba.

Biassoni, C. (2013). Curriculum Vitae. Universidad Tecnológica Nacional. Facultad Regional Córdoba. Mimeo.

Blalock, H. (1966). Estadística social. México: Fondo de Cultura Económica.

Blanco, A. (1968). Algunos aspectos de la percepción espacial en ciegos y videntes. Tesis de licenciatura no publicada. Córdoba: Universidad Nacional de Córdoba.

Brock, A. (2014). What is a polycentric history of psychology? Estudos e Pesquisas em Psicologia, 14(2), 646-659.

Bryan, M. E., Tempest, W. \& Barbenza, C. (1969). Individual loudness functions. International Audiology. 8(1), 113-119.

Buchbinder, P. (2005). Historia de las universidades argentinas. Buenos Aires: Sudamericana.

Carpintero, H., Ferrándiz, A. \& Lafuente, E. (1997). Juan Cuatrecasas y su visión psicológica de hombre. Cuadernos Argentinos de Historia de la Psicología, 3(1/2), 11-22.

Castro de Ortiz $(\mathrm{s} / \mathrm{f})$. Determinación de la relación entre tempo y edad cronológica. Tesis de licenciatura no publicada. Córdoba: Universidad Nacional de Córdoba.

Chauchard, P. (1953) Los mensajes de nuestros sentidos. Barcelona: Salvat. 
Consejo Nacional de Investigaciones Científicas y Técnicas ( $\mathrm{s} / \mathrm{f}$ ). Legajo del Ingeniero Guillermo Fuchs. Departamento de Personal del CONICET. Mimeo.

Cornachione, M.A. (1965). Determinación de la relación entre tempo y edad cronológica. Tesis de licenciatura no publicada. Córdoba: Universidad Nacional de Córdoba.

Cortada, N. (2008). Autobiografia. Mimeo. Recuperado de http://www.uba.ar/download/profesores_premiados/ncortadaau tobio. pdf, 22/06/15.

Cuatrecasas, J. (1963) El hombre, animal óptico. Buenos Aires: Eudeba.

Delgado, H. \& Ibérico, M. (1966). Psicología. Barcelona: Ed. Científico-Médica.

Detrixhe, M. \& Papini, M. (1978). Enrique Mouchet: 1886-1977. In memoriam. Revista Latinoamericana de Psicología, 10(2), 295295.

Falcone, R. (2012). Psicología en Argentina: impronta europea y carácter nacional. Revista Interamericana de Psicología, 46(1), 87-98.

Fernández, A. (1967). Estudio de las respuestas de un grupo de estudiantes universitarios frente a estímulos sonoros distorsionados. Tesis de licenciatura no publicada. Córdoba: Universidad Nacional de Córdoba.

Ferrari, F. J. (2013). Virgilio Ducceschi y el primer laboratorio de psicología experimental en Córdoba, Argentina (1907). Estudos e Pesquisas em Psicologia, 13(2), 779-805.

Foschi, M. (1968). Algunos aspectos de la percepción espacial en ciegos y videntes. Tesis de licenciatura no publicada. Córdoba: Universidad Nacional de Córdoba.

Fuchs, G. (1958). Acústica. Córdoba: Imprenta de la Universidad Nacional de Córdoba.

Fuchs, G. (1966). A new Anechoic Chamber. 31 Convention of Audio Engeniering Society. USA.

Fuchs, G. (1969). El Departamento de Investigaciones Acústicas y Luminotécnicas. CIAL. Revista de Psicología. 1, 13-20.

Guirao, M. (1980). Los sentidos, bases de la percepción. Madrid: Alhambra.

Guirao, M. (1987). Estimaciones numéricas de brillo y luminosidad con y sin rangos fijos. Revista Latinoamericana de Psicología, 19(3), 297-317.

Guirao, M. (2006). Remembering S. S. Stevens: the man, the teacher, the psychophysicist. Roundtable celebrating S. S. Stevens organised by Scharf, On Fechner's Day 2006 at the University Of Hertfordshire. En Informe del Laboratorio de Investigaciones Sensoriales 2006. Retirado de 
http://www.lis.secyt. gov.ar/prev/Institucional/fechner\%202006 .htm el 22/06/15.

Guirao, M. (2008). Mi experiencia en la carrera científica: Historia de un proyecto pionero. En Mujeres de la ciencia: dos continentes. Encuentro Hispano-Argentino de Científicas. Buenos Aires, Argentina.

Guirao, M. \& Valciukas, J. A. (1975). Perceived Vibration and the Loudness of Low Frequency Tones. Perception \& Psychophysics, $17(5), 460-464$.

Houssay, B. (1955) Fisiología humana. Buenos Aires: El Ateneo.

Hurtado, D. (2010). La ciencia argentina. Un proyecto inconcluso: 1930-2000. Buenos Aires: Edhasa.

Klappenbach, H. (2001). Dos editoriales en los comienzos de la profesionalización de la psicología en Argentina. Memorándum, Belo Horizonte, 1, 61-71.

Klappenbach, H. (2006). Periodización de la psicología en Argentina. Revista de Historia de la Psicología, 27(1), 109-164.

Laboratorio de Investigaciones Sensoriales (1978). Informe de los diez primeros años. Buenos Aires: CONICET.

Mc Robert, H., Bryan, M. E. \& Tempest, W. (1965). Magnitude estimation of loudness. J ournal of Sound and Vibration, 2, 391.

Merleau Ponty, M. (1957). Fenomenología de la Percepción. México: Fondo de Cultura Económica.

Mira y Lopez, E. (1952). Psiquiatría. Buenos Aires: El Ateneo.

Murat, F., Verzini de Romera, A. M., Biassoni de Serra, E. C. \& Fuchs, G. (1979). After-Effects of Exposure to a High-Intensity Noise. Acústica, 42(4), 270-273.

Orrico de Stuart, A. M. (1966). Privacidad y Mediciones subjetivas. Tesis de licenciatura no publicada. Córdoba: Universidad Nacional de Córdoba.

Papini, M. (1976). Datos para una historia de la psicología experimental argentina (hasta 1930). Revista Latinoamericana de Psicología, 8(2), 319-335.

Piaget, J. (1960). Psicología de la Inteligencia. Buenos Aires: Psique.

Piaget, J. \& Inhelder, B. (1947). La representation de I'espace chez I'enfant. Paris: P.U.F.

Piéron, H. (1960). La sensación. Buenos Aires: Paidós.

Piéron, H. (1964). Lexicon Kapelusz Psicología. Buenos Aires: Kapelusz.

Piñeda, M. A. \& Jacó-Vilela, M. A. (2014). Ciencia psicológica y profesionalización en Argentina y Brasil: 1930-1980. Universitas Psychologica, 13(5), 2015-2033.

Rossi, L., Falcone, R., \& Ibarra, F. (2014). Emilio Mira y López en Argentina. Revista de Historia de la Psicología, 35(2), 93-110. 
Rossi, L., Ibarra, M. F., \& Kirsch, U. (2010). Psicología en Argentina: criterios psicológicos en los diseños de fichas del área laboral (1920-1945). Anuario de investigaciones, 17, 401-407.

Saal, D. (1968). Distorsiones perceptivas determinadas por la orientación previa del sujeto. Un estudio evolutivo. Tesis de licenciatura no publicada. Córdoba: Universidad Nacional de Córdoba.

Saforcada, E. (1963). Estudio de las relaciones entre fuerza sonora y molestia. Tesis de licenciatura no publicada. Córdoba: Universidad Nacional de Córdoba.

Serra, M. R. (2000). Obituario. Profesor Ingeniero Guillermo Luis Fuchs. Interdisciplinaria, 17 (2). Retirado de http://www. redalyc.org/articulo.oa?id=18011322005 el 22/06/15.

Siegel, S. (1956). Non parametric statistics for the behavioral science. New York: Mc Graw Hill.

Stevens, J. C. \& Guirao, M. (1964). Individual loudness functions. Journal of the Acoustical Society of America, 36, 2210.

Stevens, S. S. \& Guirao, M. (1963). Subjective Scaling of Length and Area and the Matching of Length to Loudness and Brightness. Journal of Experimental Psychology, 66, 177-186.

Stevens, S. S. \& Guirao, M. (1967). Loudness Functions under Inhibition. Perception \& Psychophysics, 2, 459-465.

Stevens, S. S., Guirao, M. \& Slawson, A. W. (1965). Loudness is a product of density times volume. Journal of Experimental Psychology, 69, 503-510.

Talak, A. M. (2000). Los primeros desarrollos académicos de la psicología en la Argentina: 1896-1919. En J. C. Ríos, R. Ruiz, J. C. Stagnaro, P. Weissmann. Psiquiatría, Psicología y Psicoanálisis: historia y memoria. (pp. 215-230). Buenos Aires: Polemos.

Teeghtsoonian, R. (2001). Stevens Stanley Smith (1906-1973). In Neil J. Smelser and Paul B. Baltes (Eds). International Enciclopedia of the Social and Behavioral Sciences. Oxford: Elsevier Science Ltd.

Triarhou, L. \& Del Cerro, M. (2006). Semicentennial tribute to the ingenious neurobiologist Christfried Jakob (1866-1956). European Neurology, 56, 176-188.

Trotanesky, S. (1965). Influencias del ruido en el tiempo de reacción. Tesis de licenciatura no publicada. Córdoba: Universidad Nacional de Córdoba.

Universidad Nacional de Cuyo (1969). Resolución de la Facultad de Ciencias de la Universidad Nacional de Cuyo N 1072.

Universidad Nacional del Litoral. (1960a). Programa de Psicología Aplicada. Mimeo. 
Universidad Nacional del Litoral. (1960b). Orientación y Selección Profesional. Mimeo.

Universidad Nacional de San Luis. (1980). Ordenanza del Rectorado № 05/1980.

Universidad Nacional de San Luis. (1982). Proyectos aprobados por la Secretaría de Ciencia y Tecnología. Memorias de la Universidad de San Luis. Mimeo.

Verzini, A. M. (1968). Análisis de la relación entre la asociación de palabras y la inteligibilidad en disílabos sin sentido del idioma castellano según un enfoque psicolingüístico, Tesis de licenciatura no publicada. Córdoba Universidad Nacional de Córdoba.

Verzini de Romera, A. M. \& Suárez de Bonet, M. C. (1985). Análisis factorial de una escala de actitudes hacia el ruido. Interdisciplinaria, 6 (1), 51-70.

Verzini de Romera, A. M., Suárez de Bonet, M. C. \& Biassoni de Serra, E. C. (1983). Construcción de escalas semánticas para la evaluación subjetiva del ruido. Acta Psiquiátrica y Psicológica de América Latina, 29, 301-306.

Vezzetti, H. (1988). El nacimiento de la psicología en la Argentina. Buenos Aires: Puntosur.

Woodworth, R. \& Schlosberg, H. (1964). Psicología Experimental. Buenos Aires: Eudeba.

Yuzuk, J. (1967). Análisis de ruidos subjetivos, rendimiento y luminancia en espectáculos visuales. Tesis de licenciatura no publicada. Córdoba: Universidad Nacional de Córdoba.

\section{Endereço para correspondência \\ María Andrea Piñeda}

Consejo Nacional de Investigaciones Científicas y Técnicas (CONICET)

Universidad Nacional de San Luis (LICIC-FaPsi-UNSL)

Laboratorio de Investigaciones en Ciencias del Comportamiento, Facultad de Psicología

Av. Ejército de los Andes, 1520, 542664424027 int. 4120, San Luis, Argentina

Endereço eletrônico: mapineda@unsl.edu.ar

\section{Patricia Scherman}

Universidad Nacional de Córdoba

Museo de Psicología - Facultad de Psicología

Av. Enrique Barros, s/n., Ciudad Universitaria, Córdoba, Argentina

Endereço eletrônico: patoscherman@gmail.com

Recebido em: 16/09/2015

Reformulado em: 09/12/2015

Aceito para publicação em: 09/01/2016

\section{Notas}

* Doctora en Psicología.

** Doctora en Psicología. 